\title{
Assessment of the Antibiotics and Other Drug Prescribing Patterns in a Primary Health Care Setting
}

\author{
$\underline{\text { Raja D' }}$, Sasithra ${ }^{2}$
}

${ }^{1}$ Professor, ${ }^{2}$ Post Graduate, Department of Community Medicine, Chettinad Hospital \& Research Institute, Chettinad Academy of Research \& Education, Kelambakkam, Chengalpattu district, Tamil Nadu, India.

DOI: https://doi.org/10.24321/0019.5138.202176

I $\mathbf{N} \mathbf{F} \mathbf{O}$

\section{Corresponding Author:}

Raja D, Department of Community Medicine, Chettinad Hospital \& Research Institute, Chettinad Academy of Research \& Education, Kelambakkam, Chengalpattu district, Tamil Nadu, India.

E-mail Id:

rajadanasekaran@gmail.com

Orcid Id:

https://orcid.org/0000-0002-4571-0407

How to cite this article:

Raja D, Sasithra S. Assessment of the Antibiotics and Other Drug Prescribing Patterns in a Primary Health Care Setting. J Commun Dis. 2021;53(4):70-75.

Date of Submission: 2021-10-30

Date of Acceptance: 2021-11-24

\section{$\begin{array}{llllllll}\mathbf{A} & \mathbf{B} & \mathbf{S} & \mathbf{T} & \mathbf{R} & \mathbf{A} & \mathbf{C} & \mathbf{T}\end{array}$}

Introduction: Appropriate drug use is critical in lowering global morbidity and death. Prescribing indicators are useful for quantitatively analysing critical aspects of prescribing practice, as well as regulating rationality in drug therapy and resource usage. Hence this study was aimed at assessing the drug prescribing pattern using WHO prescribing indicators at the primary health care level.

Methods: A cross-sectional study was conducted during the first 2 weeks of July 2021 in the rural health centre of a tertiary care hospital in Chengalpattu, Tamil Nadu. All prescription copies from the outpatient pharmacy during the study period were collected and analysed using WHO prescribing indicators to assess the rational use of drugs. Prescriptions containing ineligible handwriting were excluded. The data collected from 340 prescriptions were analysed using IBM-SPSS, v21.0. Quantitative variables were described in terms of percentage.

Results: Per prescription, the average number of medications was 3.7. Antibiotics and injections prescribed were $32.9 \%$ and $30.6 \%$ respectively. The most commonly given antibiotics were amoxicillin (23.9\%), azithromycin (4.4\%), and metronidazole (3.9\%). The percentages of medications prescribed by generic name and from the essential pharmaceuticals list, respectively, were $80.3 \%$ and $100 \%$.

Conclusion: This deviation from normal standards reveals that unnecessary drug use is still obvious in actual clinical practice and persists as a public health challenge. Prescription standards should be improved among health professionals and the negative consequences of inappropriate drug and antibiotics prescription should be explained.

Keywords: Prescribing Pattern, Antibiotics, Prescribing Indicators, Rational Drug Use, Polypharmacy 


\section{Introduction}

The World Health Organization has described rational use of drugs as "patients receive medications appropriate to their clinical needs, in doses that meet their own individual requirements, for an adequate period of time, and at the lowest cost to them and their community". ${ }^{1}$ Rational drug use is also defined as "a right drug, at the right time, in right dose, to the right patient, in right strength, right administration, and with a right cost". ${ }^{2}$

Medicines are crucial in the delivery of healthcare services all around the world. In India, for example, there are approximately 70,000 drugs in use. ${ }^{3}$ According to World Health Organization research, around half of all the drugs are wrongly administered, disseminated, or marketed, and more than half of all afflicted persons fail to use prescriptions as recommended or supplied. ${ }^{4}$ Such inefficient use wastes resources and harms patients by resulting in unsatisfactory outcomes, significant side effects, and growing antibiotic resistance (AMR). AMR is one amid the top ten emerging global health and development threats which necessitate continuous surveillance of antimicrobial use throughout all health sectors. ${ }^{5}$

Utilisation of antibiotics has enlarged worldwide with a greater portion arising from middle- and low-income countries. ${ }^{6}$ Furthermore, the association between enhanced misuse of antibiotics to the development and distribution of resistant microorganisms has been established by many studies. Adding to it, the Global Antimicrobial Resistance and Use Surveillance System's (GLASS) recent report has illustrated that the resistance rate of ciprofloxacin ranged from $8.4 \%$ to $92.9 \%$ for Escherichia coli and from $4.1 \%$ to $79.4 \%$ for Klebsiella pneumoniae. ${ }^{7}$ Deprived of antimicrobials effective action, the achievement of modern medicine in treating various communicable and non-communicable diseases would be at greater risk.

Furthermore, the quality of a prescription reflects a physician's ability as well as their perspective on logical prescribing. However, according to a systematic review, medication errors can affect anywhere from 4.2 to $82 \%$ of prescriptions. ${ }^{8}$ WHO developed and verified the core drug use indicators, a simple and rapid technique for assessing prescribing patterns on a quantitative basis, to help alleviate these issues. Furthermore, a study of prescribing behaviour is necessary to evaluate rational drug use and optimal resource utilisation. Appraising drug prescribing patterns offers advantageous feedback to prescribers to improve their prescriptions and help the policymakers in setting priorities to endorse the rational use of medicines nationwide.

\section{Materials and Method}

A cross-sectional study was conducted in the rural health centre of a tertiary care hospital in Chengalpattu, Tamil
Nadu. The rural field practising area is situated $25 \mathrm{kms}$ away from the tertiary care hospital. It covers 12 villages under two blocks with a total population of 39,545 (the Census 2011). ${ }^{9}$ The study was conducted during the first two weeks of July 2021. Based on the study by Ghosh A, ${ }^{10}$ considering $23 \%$ prevalence of antimicrobial prescribing pattern at $95 \%$ confidence interval and allowable error $5 \%$, the sample size was calculated to be 283 . Assuming a $20 \%$ non-response rate, the final sample size was rounded off to 340 prescriptions.

A copy of all prescriptions issued from various outpatient departments during the first two weeks of July 2021 from the rural health centre was collected. Through simple random sampling technique, the required 340 study prescriptions were selected out of the 728 prescriptions issued during the study period through computer-generated random numbers. Prescriptions that contained patient-related information, medication-related information, provisional/ final diagnosis mentioned were included in the study. Those prescriptions with illegible handwriting and prescriptions from the same patient ID collected during multiple visits were excluded from the study.

Data were collected using WHO prescribing indicators to assess rational use of drugs from those prescriptions that met the predefined inclusion and exclusion criteria. Sociodemographic details such as gender and age of the patients were collected from the prescription. Other details collected from the prescriptions were provisional diagnosis, number of drugs prescribed, number of injections prescribed, and names of the drugs prescribed.

While calculating the number of drugs prescribed, fixed combination drugs were counted as one item only according to the WHO core drug use prescribing indicators. Among the injections category, injections in the form of immunisation were excluded. The data collected were entered in Microsoft Excel spreadsheet and analysed using IBM-SPSS-v21.0. Quantitative variables were described in terms of frequency and percentage. The study was approved by the Institutional Human Ethics Committee. The operational standards used in the study are as follows:

\section{WHO Reference Standards for Prescribing Indicators"}

- Percentage of prescriptions containing generic drug $-100 \%$

- Percentage of drugs prescribed from Essential Medical List (EML) - 100\%

- Average number of drugs per prescription - 1.6 to 1.8

- Percentage of antibiotic prescribed - 20.0 to $26.8 \%$

- Percentage of injectable prescribed - 13.4 to $24.1 \%$

Results

Table 1 shows the socio-demographic distribution of study 
prescriptions. Majority of the prescriptions belonged to the age group of $31-60$ years (37.7\%) and by gender classification, $51.8 \%$ were prescriptions of male patients followed by $48.2 \%$ female patients' prescriptions.

Table I.Socio-demographic Distribution $(\mathbf{N}=340)$

\begin{tabular}{|c|c|}
\hline Characteristics & $\mathbf{n ~ ( \% )}$ \\
\hline Age (years) & \\
\hline$<18$ & $46(13.5)$ \\
\hline $18-30$ & $104(30.6)$ \\
\hline $31-60$ & $128(37.7)$ \\
\hline$>60$ & $62(18.2)$ \\
\hline Gender & \\
\hline Male & $176(51.8)$ \\
\hline Female & $164(48.2)$ \\
\hline
\end{tabular}

$52.6 \%$ of the prescriptions had prescribed four to six drugs followed by $44.4 \%$ with less than three drugs prescribed per prescription as shown in the summary of drugs per encounter table (Table 2 ).

Table 2.Summary of Drugs per Encounter $(\mathbf{N}=340)$

\begin{tabular}{|c|c|}
\hline Number of Drugs per Encounter & $\mathbf{n}(\%)$ \\
\hline$<3$ drugs & $151(44.4)$ \\
\hline $4-6$ drugs & $179(52.6)$ \\
\hline$>6$ drugs & $10(3)$ \\
\hline
\end{tabular}

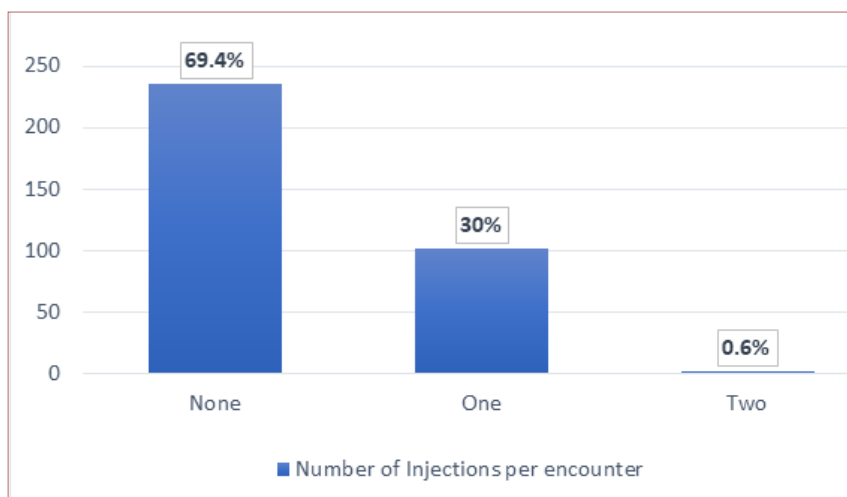

Figure I.Summary of Injections per Encounter $(\mathbf{N}=\mathbf{3 4 0})$ The bar chart in Figure 1 represents the summary data of injections prescribed per prescription. Majority (69.4\%) of the prescriptions had no injections prescribed in them.

The pie chart represents the number of antimicrobials prescribed per prescription. $65 \%$ of the prescriptions had no antimicrobials prescribed followed by prescriptions with one antimicrobial prescribed (32\%) (Figure 2).

Table 3 depicts the most common diagnoses encountered in the prescriptions. Majority of the study prescriptions had provisional diagnoses pertaining to the musculoskeletal system (35.3\%) followed by provisional diagnoses associated with the respiratory system (22.1\%) and so on.

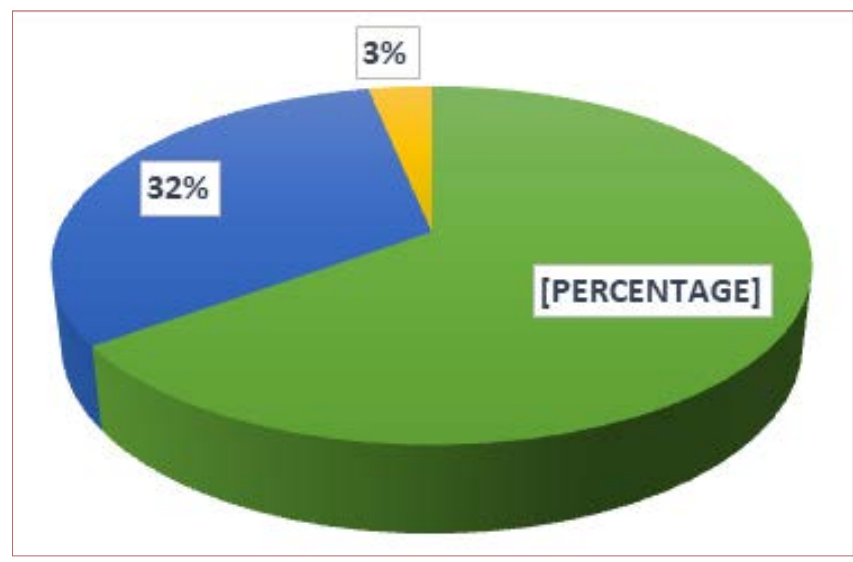

Figure 2.Summary of Antimicrobials per Encounter $(\mathbf{N}=340)$

Table 3.Most Common Provisional Diagnoses encountered in Prescriptions ( $N=340)$

\begin{tabular}{|c|c|}
\hline Diagnosis & $\mathbf{n}(\%)$ \\
\hline Disease of musculoskeletal system & $120(35.3)$ \\
\hline Disease of respiratory system & $75(22.1)$ \\
\hline Disease of endocrine system & $42(12.4)$ \\
\hline Disease of gastrointestinal system & $30(8.8)$ \\
\hline Infectious disease & $22(6.5)$ \\
\hline Dental disease & $16(4.7)$ \\
\hline Disease of genitourinary system & $11(3.2)$ \\
\hline Disease of skin & $10(2.9)$ \\
\hline Disease of blood cells & $9(2.6)$ \\
\hline Disease of eye & $5(1.5)$ \\
\hline
\end{tabular}

Among the antimicrobials prescribed in the rural health centre, the penicillin group of drugs $(21.5 \%)$ were the most commonly prescribed antimicrobial followed by the macrolide group (3.8\%) and fluoroquinolones (3.5\%) as presented in Table 4.

Table 4.Most Commonly Prescribed Antimicrobials $(\mathbf{N}=340)$

\begin{tabular}{|c|c|}
\hline Antimicrobial Groups & $\mathbf{n ~ ( \% )}$ \\
\hline Penicillin & $73(21.5)$ \\
\hline Macrolides & $13(3.8)$ \\
\hline Fluoroquinolones & $12(3.5)$ \\
\hline Nitroimidazole & $11(3.2)$ \\
\hline Antifungal & $11(3.2)$ \\
\hline Aminoglycosides & $2(0.6)$ \\
\hline Sulphonamides & $1(0.3)$ \\
\hline
\end{tabular}

Table 5 displays the observed prescribing indicators against the WHO standard values. The average number of drugs 
per encounter was higher in the rural health centre (3.7) when compared to the standards. The percentages of encounters with antibiotics and injections were $32.9 \%$ and $30.6 \%$ respectively. $80.3 \%$ of drugs were being prescribed by generic name in the rural health centre. $100 \%$ of the prescribed drugs were from the essential drug list (National List of Essential Medicines, 2015)..$^{12}$
Our current study has $30.6 \%$ of prescriptions with injections prescribed which was higher when compared to Aravamuthan A et al.'s similar study in Tamil $\mathrm{Nadu}^{3}$ (24.3\%) and Shelet PR et al.'s study in Western India $(20.8 \%)^{14}$. Yimenu et al.'s study had only $6.3 \%$ of injections prescribed which was lower than the WHO reference range of $13.4 \%$ to $24.1 \%{ }^{6}$ Injection formulations were known

Table 5.Summary Report using WHO Prescribing Indicators

\begin{tabular}{|c|c|c|c|c|}
\hline Prescribing Indicators & Total $(\mathbf{n})$ & Denominator (N) & Average & WHO Standard Value \\
\hline $\begin{array}{c}\text { Average number of drugs per } \\
\text { encounter }\end{array}$ & 1261 & 340 & 3.7 & $1.6-1.8$ \\
\hline Prescribing indicators & Total $(\mathbf{n})$ & Denominator (N) & Percentage & WHO Standard Value \\
\hline $\begin{array}{c}\text { Percentage of encounters with } \\
\text { antibiotics }\end{array}$ & 112 & 340 & 32.9 & $20-26.8$ \\
\hline $\begin{array}{c}\text { Percentage of encounters with } \\
\text { injections }\end{array}$ & 104 & 340 & 30.6 & $13.4-24.1$ \\
\hline $\begin{array}{c}\text { Percentage of drugs prescribed by } \\
\text { generic name }\end{array}$ & 1012 & 1261 & 80.3 & 100 \\
\hline $\begin{array}{c}\text { Percentage of drugs prescribed } \\
\text { from essential drug list }\end{array}$ & 1261 & 1261 & 100 & 100 \\
\hline
\end{tabular}

\section{Discussion}

The results of the current study revealed that all (100\%) the randomly selected study prescriptions had the necessary patient identification details such as age and gender mentioned in it. In this study's drug-related parameters, all prescriptions $(100 \%)$ had the right name, dose form, strength, frequency, and duration of the medications prescribed. Similar studies conducted in Lahore had only $55 \%, 42 \%$, and $87 \%$ of prescriptions with age, gender, and name (identity) of patient mentioned in it. ${ }^{13}$

Citing the patient identification details is obligatory as it can avoid unnecessary mishandling of the prescribed drugs to the wrong person and it also aids in prescribing the correct drug dosage according to the patient's age. In Shelat et al.'s study, patients name was mentioned in all prescriptions $(100 \%)$ whereas age, gender-related details were not found in any of the study prescriptions. ${ }^{14}$

The average number of drugs prescribed per prescription was 3.7 in the present study whereas the WHO reference standard varies between 1.6 to 1.8 . A study conducted by Patel DJ et al. in Gujarat, India showed an average of 9.4 which was higher when compared to our study and also the WHO standards. ${ }^{15}$ This increase in the number of drugs per prescription clearly indicates the lack of competency of the prescribers, increasing polypharmacy practice and lack of properly established treatment guidelines. Yimenu et al. conducted a similar study in Ethiopia where the average drugs prescribed per prescription was found to be 1.6 which was consistent with the WHO standards. ${ }^{6}$ for rapid symptomatic benefits and they are sometimes being prescribed on request by the patients. However, an increase in the number of injections might add extra financial burden among the patients and hence injections must be prescribed only on a necessity basis.

The percentage of encounters with antibiotics was $32.9 \%$ in the present study which was found to be higher than the reference range of $20 \%-26.8 \%$. A similar study by Desalegn in Ethiopia had $58 \%$ of antibiotics prescribed. ${ }^{1} \mathrm{~A}$ study by Shelet PR et al. in Western India had $53.6 \%$ and another by Otaam et al. in Jordan had $60.9 \%$ of antibiotics prescribed. ${ }^{14,16}$ This increased and inappropriate usage of antibiotics will lead to the early development of drug resistance among the general population thereby making it difficult to treat the infections.

Among the various antibiotics prescribed, the penicillin group (21.5\%) followed by the macrolide group of drugs (3.8\%) had a higher frequency when compared to other drugs prescribed in the present study which coincided with Yimenu et al.'s study (38.5\% and 15\% respectively). ${ }^{6} \mathrm{~A}$ similar study by Wang et al. showed that the cephalosporin group of drugs (47\%) were being prescribed commonly followed by the macrolide group (21\%) in OPDs of China. ${ }^{17}$

Nearly $80.3 \%$ of the drugs were prescribed by their generic name in the present study whereas only $0.5 \%, 3 \%$ and $5 \%$ of drugs were prescribed by their generic names in Babar HS et al.'s, Patel DJ et al.'s and Otaam et al.'s studies respectively. ${ }^{13,14,15}$ These variations would have existed because of the differences in the country's medication 
purchasing pattern, prescribing standards followed, and prescribers' choice of drug brand selection.

In the current study, all the drugs dispensed for the study prescriptions were present in the National essential drug list $(100 \%)$ which also coincides with the WHO reference standards. This denotes that good prescribing practices and strict pharmaceutical policies are being followed in the rural health centre. Various studies had $28.4 \%, 95.3 \%$, $93 \%$ and $96.6 \%$ of drugs being prescribed from the essential drug list. ${ }^{1,13,16}$

\section{Conclusion}

The current study's drug prescribing pattern demonstrated variations from WHO recommendations. This deviation has a great effect on the consequences of the patient's health condition and hence periodical evaluation and necessary interventions should be implemented to make sure that drugs are being used prudently. Further deviations can be managed by refining the standards of prescription in possible ways such as training and updating the clinicians by organising regular continuing education programmes, conferences and so on.

The clinicians can also be instructed to have their own copy of the standard treatment guidelines, essential drug list and antibiotic policies being followed. Besides, improving the prescription standards, professionals should also be enlightened about the negative consequences of inappropriate drug and antibiotics use. At the hospital level, programmes to improve systematic monitoring of antibiotic use by means of organising periodic prescriptions reviews and providing feedback to improve the quality of antimicrobials usage and to rationalise drug use are suggested.

\section{Source of Funding: None Conflict of Interest: None \\ References}

1. Desalegn AA. Assessment of drug use pattern using WHO prescribing indicators at Hawassa University teaching and referral hospital, south Ethiopia: a crosssectional study. BMC Health Serv Res. 2013 May;13:170. [PubMed] [Google Scholar]

2. Amaha N, Weldemariam D, Abdu N, Tesfamariam E. Prescribing practices using WHO prescribing indicators and factors associated with antibiotic prescribing in six community pharmacies in Asmara, Eritrea: a crosssectional study. Antimicrob Resist Infect Control. 2019 Oct;8:163. [PubMed] [Google Scholar]

3. Aravamuthan A, Arputhavanan M, Subramaniam K, Udaya Chander JSJ. Assessment of current prescribing practices using World Health Organization core drug use and complementary indicators in selected rural community pharmacies in Southern India. J Pharm Policy Pract. 2017 Jul;10:1. [PubMed] [Google Scholar]

4. Mathew R, Sayyed H, Behera S, Maleki K, Pawar S. Evaluation of antibiotic prescribing pattern in pediatrics in a tertiary care hospital. Avicenna J Med. 2021 Jan;11(1):15-9. [PubMed] [Google Scholar]

5. World Health Organization [Internet]. Ten health issues WHO will tackle this year; [cited 2021 Oct 27]. Available from: https://www.who.int/news-room/spotlight/tenthreats-to-global-health-in-2019

6. Yimenu D, Emam A, Elemineh E, Atalay W. Assessment of antibiotic prescribing patterns at outpatient pharmacy using World Health Organization prescribing indicators. J Prim Care Community Health. 2019 Jan-Dec;10:2150132719886942. [PubMed] [Google Scholar]

7. World Health Organization [Internet]. Antimicrobial resistance; [cited 2021 Oct 27]. Available from: https://www.who.int/news-room/fact-sheets/detail/ antimicrobial-resistance

8. Ahsan M, Shaifali DI, Khurram Mallick DA, Singh HK, Verma S, Shekhar A. Prescription auditing based on World Health Organization (WHO) prescribing indicators in a teaching hospital in North India. Int J Med Res Rev. 2016;4(10):1847-52. [Google Scholar]

9. Tamil Nadu Population Sex Ratio in Tamil Nadu Literacy rate data 2011-2021 [Internet]. Census2011.co.in. 2021 [cited 5 December 2021]. Available from: https://www. census2011.co.in/census/state/tamil+nadu.html

10. Ghosh A, Adhikari A, Indu R, Ghosh S, Banik S, Paul S. A comparative study of drug prescribing indicators in various hospitals of West Bengal, India. Int J Res Med Sci. 2018;6:2063-7. [Google Scholar]

11. World Health Organization [Internet]. Action programme on essential drugs and vaccines. How to investigate drug use in health facilities: selected drug use indicators; 1993 [cited 2021 Nov 17]. Available from: https://apps.who.int/iris/handle/10665/60519

12. National List of Essential Medicine [Internet]. 2021 [cited 5 December 2021]. Available from: https://www. nhp.gov.in/NHPfiles/NLEM\%2c\%202015.pdf

13. Babar HS, Hussain S, Maqsood Z, Dad H, Khan M, Rahman AA, Bukhsh A. Adherence to prescription format and compliance with who core prescribing indicators. J Pharm Sci Res. 2014;6(4):195-9. [Google Scholar]

14. Shelat PR, Kumbar SK. Analysis of out door patients' prescriptions according to World Health Organization (WHO) prescribing indicators among private hospitals in Western India. J Clin Diagn Res. 2015 Mar;9(3):FC01-4. [PubMed] [Google Scholar]

15. Patel DJ, Deshpande DS. Assessment of antibiotic prescribing pattern using World Health Organization 
prescribing indicators in a tertiary care hospital, Gujarat. Int J Pharma Sci Inv. 2020;9(4):17-22. [Google Scholar]

16. World Health Organization [Internet]. Evaluation of drug use in Jordan using WHO prescribing indicators; 2002 [cited 2021 Oct 25]. Available from: https://apps. who.int/iris/handle/10665/119197

17. Wang YY, Du P, Huang F, Li DJ, Gu J, Shen FM, Jiang YY. Antimicrobial prescribing patterns in a large tertiary hospital in Shanghai, China. Int J Antimicrob Agents. 2016 Dec;48(6):666-73. [PubMed] [Google Scholar] 